\title{
FISHING IN TIDAL STREAMS: NEW RADIAL VELOCITY AND PROPER MOTION CONSTRAINTS ON THE ORBIT OF THE ANTICENTER STREAM
}

\author{
C. J. GRILlmaiR ${ }^{1,2}$ \\ Spitzer Science Center, 1200 East California Boulevard, Pasadena, CA 91125; carl@ipac.caltech.edu \\ AND \\ JefFrey L. Carlin and Steven R. Majewski \\ Department of Astronomy, University of Virginia, P.O. Box 400325, Charlottesville, VA 22904-4325 \\ Received 2008 October 6; accepted 2008 October 30; published 2008 November 7
}

\begin{abstract}
We have obtained radial velocity measurements for stars in two widely separated fields in the Anticenter Stream. Combined with SDSS/USNO-B proper motions, the new measurements allow us to establish that the stream is on a nearly circular, somewhat inclined, prograde orbit around the Galaxy. While the orbital eccentricity is similar to that previously determined for the Monoceros stream, the sizes, inclinations, and positions of the orbits for the two systems differ significantly. Integrating our best-fitting Anticenter Stream orbit forward, we find that it is closely aligned along and lies almost on top of a streamlike feature previously designated the "Eastern Banded Structure." The position of this feature coincides with the apogalacticon of the orbit. We tentatively conclude that this feature is the next wrap of the Anticenter Stream.
\end{abstract}

Subject headings: Galaxy: halo - Galaxy: kinematics and dynamics — Galaxy: structure

\section{INTRODUCTION}

With numerous stellar streams now known to inhabit the Galactic halo (Ibata et al. 2001; Majewski et al. 2003; Yanny et al. 2003; Rocha-Pinto et al. 2004; Belokurov et al. 2006; Grillmair \& Dionatos 2006a, 2006b; Odenkirchen et al. 2001; Grillmair 2006a, 2006b, 2008) it appears that we are entering an interesting new era in which we can hope gradually to unravel the accretion events that are thought to have built up our Galaxy. As the formation and dynamical evolution of tidal streams are strongly affected by the Galactic potential, careful study of their positions and motions will help us to refine our knowledge of both the global and small-scale distribution of dark matter (Murali \& Dubinski 1999; Johnston et al. 2002, 2005).

The Anticenter Stream (ACS) is one of the most visible features in stellar maps (e.g., Belokurov et al. 2007, Grillmair 2006b, hereafter G06) derived from the Sloan Digital Sky Survey (SDSS). The ACS extends for some $65^{\circ}$ along the western edge of the survey area. G06 used a matched-filter technique to examine the detailed structure of the stream. Among the findings of that work was that the stream appears to be made up of at least three separate, relatively cold streams, possibly the remnants of dynamically distinct components within the progenitor. Of particular interest was the finding that, since the ACS apparently does not itself pass through Monoceros, and since it lies some $15^{\circ}$ to the west of the best estimate of Monoceros' orbit (Penarrubia et al. 2005), the two streams must be distinct. Using the position of the stream on the sky and an estimated distance, G06 was able to put limited constraints on the orbit of the stream. The chief uncertainty in this estimate was the lack of radial velocity and proper motion data.

In this paper we make progress in alleviating this uncertainty by presenting new radial velocity measurements of stars in the

\footnotetext{
${ }^{1}$ Visiting Astronomer, Kitt Peak National Observatory, National Optical Astronomy Observatory, which is operated by the Association of Universities for Research in Astronomy (AURA) under cooperative agreement with the National Science Foundation.

${ }^{2}$ The WIYN Observatory is a joint facility of the University of WisconsinMadison, Indiana University, Yale University, and the National Optical Astronomy Observatory.
}

ACS (§ 2). We estimate stream velocities and proper motions in $\S 3$, and put new constraints on the orbit of the ACS in $\S 4$.

\section{OBSERVATIONS}

Radial velocity measurements for stars selected from the SDSS were carried out using the HYDRA multiobject spectrograph on the WIYN telescope in February 2007. Target stars were selected to have $18.6<g<20.1$ and $0.21<g-r<$ 0.43 , which places them on or near the color-magnitude sequence obtained for the ACS by G06. The sample in one field was further culled with proper motions from the Casetti-Dinescu et al. (2006) study of Selected Area (SA) 76, which lies along the ACS; stars were selected to have solar reflex-corrected proper motions consistent (to within $\pm 2 \sigma$ ) with motion along the north-south orientation of the ACS. A total of 123 stars were observed in two $50^{\prime} \times 50^{\prime}$ fields: ACS-B centered at $(\alpha, \delta)_{2000}=\left(124^{\circ}, 37.5^{\circ}\right)$, and ACS-C (SA 76) centered at $(\alpha, \delta)_{2000}=\left(125^{\circ}, 14.7^{\circ}\right)$.

We used the600@10.1 grating in first order, centered at $5400 \AA$, to give a working wavelength range of 4000-6800 $\AA$ at a dispersion of $1.397 \AA$ pixel $^{-1}$ and a spectral resolution of $3.35 \AA$. This region was selected to include the $\mathrm{H} \beta, \mathrm{Mg}$ triplet, $\mathrm{Na} \mathrm{D}$, and $\mathrm{H} \alpha$ spectral features, among others. Integration times totaled $9 \times 2400 \mathrm{~s}(6 \mathrm{hr})$ for each ACS field, although the ACS-C observations were beset by high winds and poor $\left(\sim 2.5^{\prime \prime}\right)$ seeing. Fifteen measurements of sky background were taken at random positions in each field.

The HYDRA data were reduced using the procedures described in Casetti-Dinescu et al. (2008). Computed velocity errors range from 6 to $20 \mathrm{~km} \mathrm{~s}^{-1}$, with a mean of $11.5 \mathrm{~km} \mathrm{~s}^{-1}$ for ACS-B. For ACS-C, computed errors range from 4 to 21 $\mathrm{km} \mathrm{s}^{-1}$, with a mean of $9.3 \mathrm{~km} \mathrm{~s}^{-1}$. A more detailed description of the observations will be presented in a forthcoming paper (J. L. Carlin et al. 2009, in preparation).

\section{ANALYSIS}

Figure 1 shows velocity histograms for stars in the two ACS fields. Also shown are the scaled radial velocity distributions 


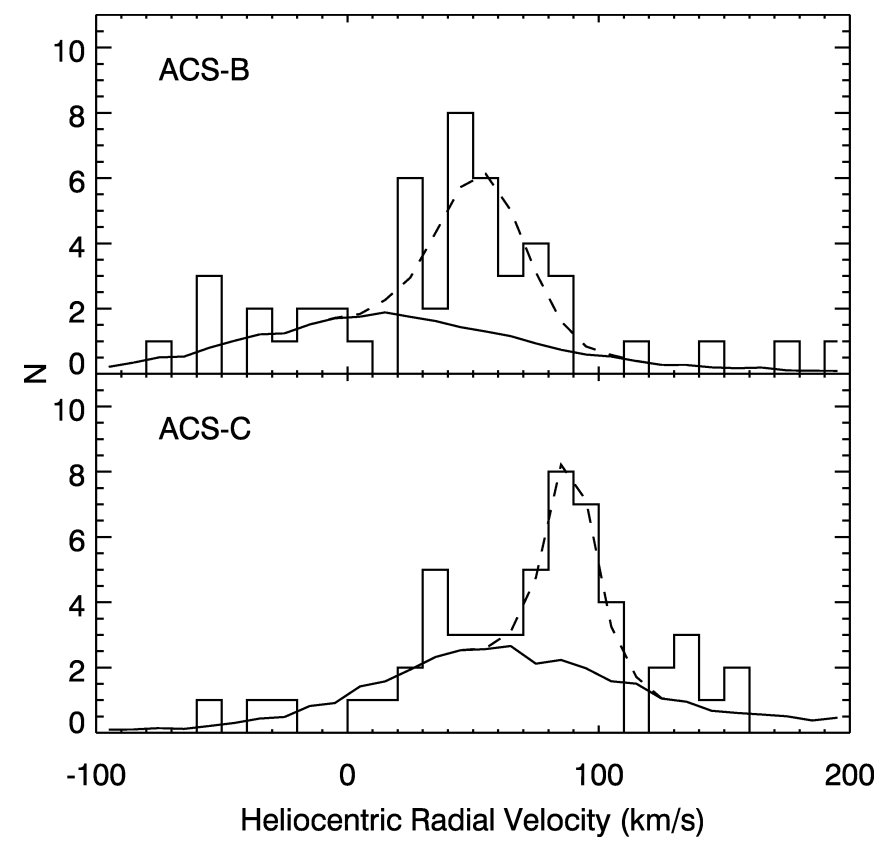

FIG. 1.-Histograms of radial velocities measured in our two fields. The smooth curves show the scaled distributions of foreground stars as predicted by the Besançon model of the Galaxy. The dashed curves show the Gaussian distributions that best fit the stream peaks. The histograms are shown using $10 \mathrm{~km} \mathrm{~s}^{-1}$ wide velocity bins, although our analysis makes use of a variety of bin widths.

(see below) predicted by the Besançon model of the Galaxy (Robin et al. 2003) in these directions over the magnitude and color ranges of our targets. The foreground distributions comprise over 10,000 realizations, and for practical purposes are free of random errors. For the restricted color and magnitude ranges applied to our targets, the predicted line-of-sight velocity dispersions of field stars are $\sim 70 \mathrm{~km} \mathrm{~s}^{-1}$.

Narrow velocity peaks are visible in both the ACS-B and ACS-C fields. We test the similarity of the observed and model distributions of radial velocities using a two-tailed Kolmogorov-Smirnoff test. For ACS-B there is a $\sim 50 \mathrm{~km} \mathrm{~s}^{-1}$ difference in the peak velocities of the two distributions, and we find that we can reject the hypothesis that the observed and predicted distributions were drawn from the same parent population at the $98.4 \%$ significance level. For ACS-C, the significance level of the null hypothesis is only $84 \%$, and we cannot rule out that the distributions were drawn from the same parent population. This is due to the higher mean velocity of foreground stars in this direction $\left(\sim 60 \mathrm{~km} \mathrm{~s}^{-1}\right)$, and to the presence of the two apparent peaks (at 35 and $90 \mathrm{~km} \mathrm{~s}^{-1}$ ) that bracket that mean velocity. The surface density of SDSS stars that match our color and magnitude selection criteria is $25 \%$ lower in the ACS$\mathrm{C}$ field than in ACS-B, but the strength of the stream (as measured from the filtered star counts of G06) is also about 35\% lower in ACS-C. As a percentage, sample contamination should therefore be similar in the two fields. Given that we observed the same total number of stars in each field, and that we observed only a fraction of the total number of stars available, we expect similar numbers of stream stars to have been selected in each field. The more pronounced ACS-C peak at $\sim 90 \mathrm{~km} \mathrm{~s}^{-1}$ is clearly at odds with the predicted foreground distribution, and the integrated number of stars in the peak (see below) approximately matches the number of stream stars in the ACS-B peak. We consequently adopt the $90 \mathrm{~km} \mathrm{~s}^{-1}$ peak as being due to ACS stars.
TABLE 1

Anticenter Stream Velocities and Proper Motions

\begin{tabular}{cccccr}
\hline \hline Field & $\begin{array}{c}V_{s} \\
\left(\mathrm{~km} \mathrm{~s}^{-1}\right)\end{array}$ & \multicolumn{1}{c}{$\begin{array}{c}\sigma_{\mathrm{s}} \\
\left(\mathrm{km} \mathrm{s}^{-1}\right)\end{array}$} & $N_{s}$ & $\begin{array}{c}\mu_{\alpha} \cos \delta \\
\left(\mathrm{mas} \mathrm{yr}^{-1}\right)\end{array}$ & \multicolumn{1}{c}{$\begin{array}{c}\mu_{\delta} \\
\left(\mathrm{mas} \mathrm{yr}^{-1}\right)\end{array}$} \\
\hline ACS-B $\ldots \ldots$ & $53.1 \pm 7.5$ & $16.1 \pm 5.9$ & 22 & $0.29 \pm 0.95$ & $-1.94 \pm 0.95$ \\
ACS-C $\ldots . .$. & $88.8 \pm 5.0$ & $6.9 \pm 3.6$ & 16 & $0.67 \pm 0.81$ & $0.73 \pm 0.80$ \\
\hline
\end{tabular}

For each field the observations are modeled as a sum of the scaled, Besançon distribution of foreground stars and a single Gaussian with mean velocity $V_{s}$, velocity dispersion $\sigma_{s}$, and number of stream stars $N_{s}$. The four fitting parameters are solved for in a least-squares sense, and the results are given in Table 1 . We use bin sizes of $3,5,10$, and $15 \mathrm{~km} \mathrm{~s}^{-1}$, beyond which the stream peaks become too undersampled to give reliable results. The fitted parameters are very nearly identical in all cases (with a dispersion of $\sim 0.8 \mathrm{~km} \mathrm{~s}^{-1}$ ), and we adopt a bin size of $5 \mathrm{~km} \mathrm{~s}^{-1}$ for the results given in Table 1. The uncertainties are estimated by constructing 1000 realizations of the data, generating Gaussian deviates for each velocity measurement using the individual measurement errors, and rebinning the results. The velocity bin walls are also randomly offset by from 1 to $4 \mathrm{~km} \mathrm{~s}^{-1}$, respectively. The uncertainties are estimated by measuring the standard deviations in the resulting best-fit parameters.

The observed stream star velocity dispersions are the convolution of the stellar velocity dispersions and the uncertainties in the measurements. Correcting the measured velocity dispersions by quadrature subtraction of the mean uncertainties in each field, we find stellar velocity dispersions of 14.9 and $5.9 \mathrm{~km}^{-1}$ for ACS-B and ACS-C, respectively. The latter is consistent with a very cold stream and suggests that we are primarily sampling one of the nearly parallel substreams or "tributaries" found by G06. The dispersion in the ACS-B field may indicate that we are measuring stars in more than one tributary or more than one orbital wrap (see below).

All of our target stars also have proper motion measurements based on a SDSS-USNO-B positional comparison (Monet et al. 2003; Munn et al. 2004). Although the individual measurement uncertainties (typically 4 mas $\mathrm{yr}^{-1}$ ) are quite sizable, averaging over a selected sample can be used to set limits on the motions of the stream. While the original SDSS proper motion catalog had small errors in the R.A. component (Ivezic et al. 2008), we use corrected proper motions kindly provided by J. Munn. We estimate the mean stream proper motion in each field by averaging over only those stars with radial velocities within $1 \sigma$ of the best-fit stream velocities. The weighted average proper motions are given in Table 1 .

\section{A NEW ORBIT FOR THE ANTICENTER STREAM}

G06 estimated the ACS to lie at a distance of $8.9 \pm 0.2 \mathrm{kpc}$. We constrain the path of the stream by selecting 30 fiducial points that trace the tributary that passes through ACS-B and ACS-C. There is some uncertainty in this procedure, because the different dynamical components of the stream are not uniformly strong along their lengths, and it is possible to confuse the different tributaries as they blend, cross one another, or fade entirely. Based on the scatter in the estimated fiducial points about a second-order polynomial fit, we assign a positional uncertainty of $0.35^{\circ}$ to each point.

Using the Galactic model of Allen \& Santillan (1991), which assumes a spherical halo potential, we generate test particle orbits and then use $\chi^{2}$ minimization to simultaneously fit the 


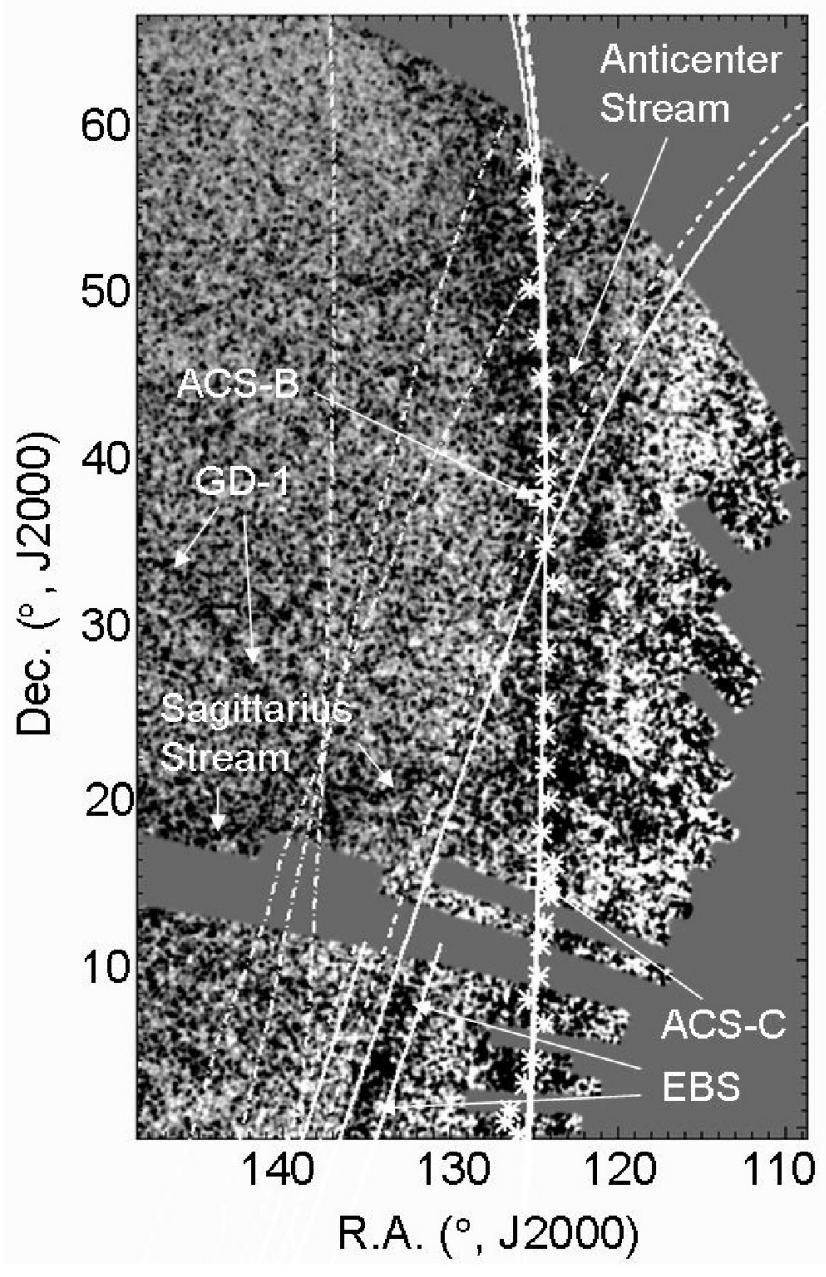

FIG. 2.-Our best-fitting orbit projections overlaid on the ACS. The underlying image is a surface density map of the western portion of the SDSS survey area, filtered for an M13-like stellar population at a distance of $9 \mathrm{kpc}$ (Grillmair 2006b). A low-order polynomial surface fit has been subtracted to remove large-scale nonuniformities, and the result has been smoothed with a Gaussian kernel with $\sigma=0.2^{\circ}$. The solid curves show the first and second wraps for model A (unconstrained by proper motions) along with their $1 \sigma$ limits in the vicinity of the EBS, while the dashed lines show orbit projections for model B (constrained using the proper motions in Table 1). The dashdotted curves show the nearby portions of the best-fitting prograde orbits for the Monoceros stream as determined by Penarrubia et al. (2005). The asterisks show the fiducial points used to constrain the position of the ACS, and the positions of ACS-B and ACS-C are indicated.

radial velocities, proper motions, distance, and the apparent path of the stream. We use a downhill simplex method, integrating orbits and comparing positions and velocities at each step. As we use 30 sky position measurements and only 2 velocity measurements, we increase the relative weight of the velocity and proper motions measurements by a factor of $30 /$ 2 in the computation of $\chi^{2}$.

In Figure 2 we show the projected paths of the best-fitting

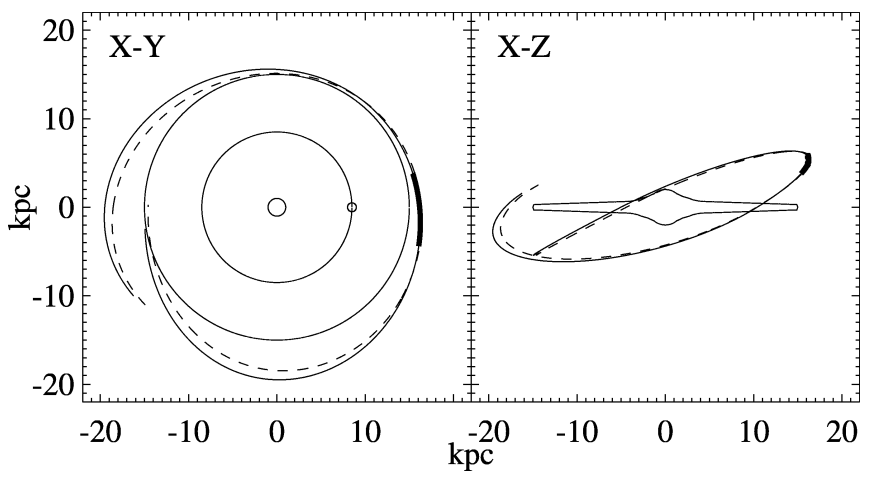

FIG. 3.-Our best-fitting orbits in right-handed, Cartesian Galactic coordinates. The Sun's position is shown as the small open circles at $R=8.5 \mathrm{kpc}$. The solid line corresponds to model A, while the dashed line shows model B. The thick portion of model A shows the section of the ACS visible in the SDSS.

prograde orbits, both with (model B) and without (model A) proper motion constraints. The $1 \sigma$ uncertainties in the integrated orbits are computed by applying Gaussian deviates to the radial velocities, proper motions, sky positions, and distance measurements using their estimated individual uncertainties. We construct 100 such realizations and recompute the bestfitting orbit in each case. The fit parameters and their estimated uncertainties are given in Table 2. The orbits are shown in Cartesian Galactic coordinates in Figure 3.

For the case where proper motions are allowed to be free parameters, the best-fit proper motions given in Table 2 lie within $1 \sigma$ of the average values in Table 1 , with the exception of $\mu_{\delta}$ for ACS-B, which lies $3 \sigma$ from its measured value. If the measured proper motions are used to constrain the fit, this departure is reduced to $1.8 \sigma$. At this point we cannot say whether the difference in $\chi^{2}$ in Table 2 is due to possible errors in the proper motion measurements or to the inappropriateness of our relatively simple Galactic potential.

Unconstrained by proper motion measurements, a retrograde

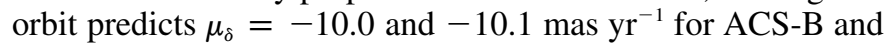
ACS-C, respectively. For stars with radial velocities within 1 $\sigma$ of the stream velocities in Table 1, only two (ACS-B) and zero (ACS-C) have $\mu_{\delta}<-10$ mas $\mathrm{yr}^{-1}$. The mean proper motions disagree with the retrograde orbit model at the 8 and 14 $\sigma$ levels for the ACS-B and ACS-C fields, respectively. We conclude with high confidence that the stars in the ACS are orbiting the Galaxy in a prograde fashion.

Our computed orbital parameters predict that apogalacticon occurs at $(\alpha, \delta)=\left(294^{\circ}, 57^{\circ}\right.$; model A) or $\left(293^{\circ}, 45^{\circ}\right.$; model B), well outside the current limits of the SDSS. On the other hand, integrating the orbits forward reveals that the subsequent apogalactica occur at $(\alpha, \delta)=\left(141^{\circ},-10.3^{\circ}\right.$; model A) or $\left(134^{\circ}, 5.1^{\circ}\right.$; model $\left.\mathrm{B}\right)$, which are within or nearly within the field shown in Figure 2. This second wrap of the orbit is closely aligned with, and lies almost on top of, the streamlike feature identified by G06 as the "Eastern Banded Structure" (EBS).

TABLE 2

Best-Fit Orbit Parameters

\begin{tabular}{|c|c|c|c|c|c|c|c|c|c|c|c|}
\hline \multirow[b]{3}{*}{ Model } & & & \multicolumn{4}{|c|}{ PROPER Motions $\left(\right.$ mas $\left.\mathrm{yr}^{-1}\right)$} & \multirow{3}{*}{$\begin{array}{r}R_{\text {peri }} \\
(\mathrm{kpc}) \\
\end{array}$} & \multirow{3}{*}{$\begin{array}{c}R_{\text {apo }} \\
(\mathrm{kpc})\end{array}$} & \multirow[b]{3}{*}{$e$} & \multirow{3}{*}{$\begin{array}{c}i \\
(\mathrm{deg}) \\
\end{array}$} & \multirow[b]{3}{*}{$\chi^{2}$} \\
\hline & \multicolumn{2}{|c|}{$V_{R}\left(\mathrm{~km} \mathrm{~s}^{-1}\right)$} & \multicolumn{2}{|c|}{ ACS-B } & \multicolumn{2}{|c|}{ ACS-C } & & & & & \\
\hline & ACS-B & ACS-C & $\mu_{\alpha} \cos \delta$ & $\mu_{\delta}$ & $\mu_{\alpha} \cos \delta$ & $\mu_{\delta}$ & & & & & \\
\hline$\ldots$ & $51.5 \pm 7.0$ & $89.1 \pm 5.5$ & $0.80 \pm 0.04$ & $-0.11 \pm 0.34$ & $0.64 \pm 0.03$ & $0.67 \pm 0.35$ & $15.5 \pm 0.9$ & $19.8 \pm 2.2$ & $0.12 \pm 0.07$ & $20.1 \pm 0.4$ & 1.2 \\
\hline B & $47.8 \pm 7.1$ & $90.3 \pm 5.2$ & $0.78 \pm 0.16$ & $-0.27 \pm 0.30$ & $0.61 \pm 0.18$ & $0.40 \pm 0.32$ & $15.4 \pm 1.1$ & $19.0 \pm 1.9$ & $0.10 \pm 0.07$ & $20.1 \pm 0.7$ & 2.3 \\
\hline
\end{tabular}


The apparent agreement between the predicted orbits and the position of the EBS is highly suggestive. The point where the two wraps cross (in projection) is predicted to occur at an R.A. of $125^{\circ}$ and declination of between $34^{\circ}$ and $37^{\circ}$, very close to the ACS-B field. Could we have inadvertently sampled stars from both the first and second wraps of the stream and could this account for the higher velocity dispersion seen in the ACS$\mathrm{B}$ field? The distance of the second wrap at this point is predicted to be $\sim 15 \mathrm{kpc}$, or about 1.1 mag fainter than the ACS itself. The turnoff and subgiant branches of the two wraps should therefore overlap, and it is certainly possible that some of our fainter targets could belong to the second wrap. The predicted radial velocity of the second wrap at this point is -15 to $-17 \mathrm{~km} \mathrm{~s}^{-1}$. While examination of Figure 1 does not reveal an obvious peak in the velocity distribution at this point, even a small number of second-wrap stars blended in with primary wrap stars would be sufficient to broaden the velocity peak. On the other hand, if we divide our observations into two roughly equal samples of stars with $g<19.6$ and $g>$ 19.6, respectively, we find that the best-fit velocity dispersion (after accounting for differing mean velocity errors) is larger for the brighter stars $\left(17 \mathrm{~km} \mathrm{~s}^{-1}\right)$ than it is for the fainter stars $\left(11 \mathrm{~km} \mathrm{~s}^{-1}\right)$. We conclude that second-wrap stars are unlikely to account for the larger velocity dispersion in ACS-B, and that the observed velocity dispersion is either intrinsic or due to the overlap of multiple tributaries in the primary wrap.

The strongest concentration of stars in the EBS occurs at $(\alpha$, $\delta)=\left(133.8^{\circ}, 3.4^{\circ}\right)$ and would be a favorable field in which to measure radial velocities for this stream. At this declination, both our models for the second wrap of the ACS predict a heliocentric radial velocity of $20 \mathrm{~km} \mathrm{~s}^{-1}$, and proper motions

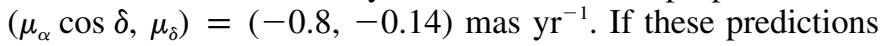
are borne out by future observations, the case for a physical association between the ACS and the EBS will be considerably strengthened.

These orbit determinations do not agree with the preliminary estimate of G06, underscoring the fact that projected position and distance alone are insufficient to provide reliable con- straints even for relatively long streams. A comparison of the orbital parameters with those of Penarrubia et al. (2005) for their best-fitting prograde orbits for the Monoceros stream reveals some interesting similarities. While these authors put the apogalacticon of Monoceros some $3 \mathrm{kpc}$ farther out and the orbital inclination some $5^{\circ}$ larger than what we find for the ACS, the orbital eccentricity they find $(0.1)$ is essentially identical to that found here. In Figure 2 we show the projected paths of the nearby portions of their best-fit prograde orbits for $q_{h}=0.6,0.7$, and 0.8 . The westernmost projection $\left(q_{h}=\right.$ 0.8 ) bears some similarity to the second wrap of the ACS, although the sky position remains offset some $8^{\circ}$ to the east. Given this near agreement, it is interesting to consider whether backward integration of the Monoceros orbit another full wrap may provide a reasonable match to the position of the ACS itself.

\section{CONCLUSIONS}

Radial velocity and proper motion measurements in two fields of the ACS show that the stream is in a prograde orbit. Integrating the orbit forward, we find that the next wrap of the ACS is aligned along and lies almost on top of the stream known as the EBS.

Further refinement of the orbit will require additional velocity measurements, more accurate proper motions, and a more realistic model of the Galactic potential. In this respect, planned spectroscopic surveys such as SEGUE2, APOGEE, and LAMOST, and proper motions from Gaia, LSST, and the SIMLite will significantly advance our understanding of this particular stream's motion and origin and, ultimately, the shape of the Galactic potential.

We are indebted to J. Munn for providing corrected SDSS-USNO-B proper motions in advance of the release of SDSS DR7. J. L. C. and S. R. M. acknowledge NSF grants AST-0307851 and AST-0807945.

Facilities: WIYN

\section{REFERENCES}

Allen, C., \& Santillan, A. 1991, Rev. Mex. AA, 22, 255

Belokurov, V., et al. 2006, ApJ, 642, L137 2007, ApJ, 658, 337

Casetti-Dinescu, D. I., Carlin, J. L., Girard, T. M., Majewski, S. R., Peñarrubia, J., \& Patterson, R. J. 2008, AJ, 135, 2013

Casetti-Dinescu, D. I., Majewski, S. R., Girard, T. M., Carlin, J. L., van Altena, W. F., Patterson, R. J., \& Law, D. R. 2006, AJ, 132, 2082

Grillmair, C. J. 2006a, ApJ, 645, L37 2006b, ApJ, 651, L29

-.2008, ApJ, submitted

Grillmair, C. J., \& Dionatos, O. 2006a, ApJ, 641, L37 . 2006b, ApJ, 643, L17

Ibata, R., Lewis, G. F., Irwin, M., Totten, E., \& Quinn, T. 2001, ApJ, 551, 294
Ivezic, Z., et al. 2008, ApJ, 684, 287

Johnston, K. V., Law, D. R., \& Majewski, S. R. 2005, ApJ, 619, 800

Johnston, K. V., Spergel, D. N., \& Hadyn, C. 2002, ApJ, 570, 656

Majewski, S. R., Skrutskie, M. F., Weinberg, M. D., \& Ostheimer, J. C. 2003, ApJ, 599, 1082

Monet, D. G., et al. 2003, AJ, 125, 984

Munn, J. A., et al. 2004, AJ, 127, 3034

Murali, C., \& Dubinski, J. 1999, AJ, 118, 911

Odenkirchen, M., et al. 2001, ApJ, 548, L165

Penarrubia, J., et al. 2005, ApJ, 626, 128

Robin, A. C., Reyle, C., Derriere, S., \& Picaud, S. 2003, A\&A, 409, 523

Rocha-Pinto, H. J., Majewski, S. R., Skrutskie, M. F., Crane, J. D., \& Patterson, R. J. 2004, ApJ, 615, 732

Yanny, B., et al. 2003, ApJ, 588, 824 